\title{
La médecine mobile et les changements qu'elle représente
}

Les opinions exprimées dans cet éditorial sont celles de l'auteur et ne représentent pas nécessairement celles de l'Association médicale canadienne ou ses filiales.

a médecine change. Nous le savons, bien sûr. Il y a de nouveaux médicaments, de nouveaux protocoles, de nouvelles marches à suivre intéressantes et des outils de formation novateurs qui font sortir la médecine de son moule traditionnel. Ce ne sont pas ces changements qui vont nous affecter le plus à l'avenir, mais bien la médecine mobile, qui est portée par une vague de changements économiques et sociaux sur le point de transformer le paysage médical. Elle a déjà commencé à se manifester avec l'adoption de la télémédecine dans les pays industrialisés. La visée de la plupart des services de médecine mobile devrait être de mieux répartir les budgets de santé, en gardant les patients en santé à domicile et en prodiguant sans tarder des soins aux malades. Mais l'objectif principal de l'adoption de la télémédecine par certains systèmes est peut-être d'accroître la visibilité de l'hôpital ou du système de santé en tant que marques offrant la télémédecine. Or, des données probantes montrent qu'elle fait augmenter le bassin de nouveaux patients, mais peut aussi faire augmenter les coûts des soins de santé, à mesure que des patients encore jamais traités viennent accaparer une partie de l'enveloppe budgétaire ${ }^{1}$. Par contre, selon d'autres observations ${ }^{2}$, la télémédecine pédiatrique aurait réduit considérablement les consultations urgentes. Cette étude a montré que sans télémédecine, $28 \%$ des parents auraient consulté aux urgences; la télémédecine a ainsi été associée à des économies projetées d'environ 114 millions de dollars pour le système de santé de la Floride².

La médecine mobile, ou virtuelle, peut avoir une dimension interactive, mais elle pourrait aussi mettre à contribution l'intelligence artificielle, les applis liées à la santé et des espaces de discussion automatisés (attention, psychiatres).

La télémédecine veut combler le désir d'améliorer la santé et l'expérience des patients, de réduire les coûts globaux, et, espérons-le, d'accroître la satisfaction des professionnels de la santé. L'attente des patients à cet égard est clairement présente. Beaucoup d'entre eux voudront sous peu que leur système de santé leur procure l'accès et la commodité qu'ils retrouvent avec les services de covoiturage ou un Apple Store. La communication par Skype avec votre médecin (ou son adjoint) deviendra monnaie courante comme outil de détection préclinique. À mon avis, aux États-Unis, c'est la crainte d'un déferlement de demandes qui empêche l'inclusion de la télémédecine dans le régime d'assurance-maladie (sauf dans les régions rurales et éloignées). Mais une fois la brèche ouverte, rien ne retiendra plus les flots, peut-être pas en ce qui concerne les demandes de consultation, mais certainement pour la prestation des soins dans divers domaines, comme la dermatologie, la psychiatrie, la médecine familiale et autres. Les systèmes de santé au Canada fonctionnent comme si cette éventualité n'existait pas - or elle risque fort bien de se matérialiser. La chirurgie pourrait demeurer à l'abri tant qu'une appli ou qu'un dispositif pour téléphone intelligent ne sera pas mis au point pour administrer une anesthésie ou pratiquer une cholécystectomie.

Pour le reste de la médecine, le mouvement « faites-le vous-même » se fraie un chemin vers les applis et les modules pour téléphones. La capacité de faire des échographies diagnostiques à domicile au moyen d'appareils connectés à un téléphone existe déjà. Les applis actuelles font tout, de la promotion du mieux-être aux diagnostics médicaux (p. ex., ulcères pédieux et érythème cutané). Avec un coup de pouce de l'intelligence artificielle, les patients pourront s'autodiagnostiquer et ils ne verront plus l'intérieur d'une clinique ou d'un hôpital à moins d'être considérés vraiment malades. Ces mouvements ne rendront pas les chirurgiens obsolètes, mais allègeront sans contredit le fardeau des autres spécialités. Nous sommes mal préparés à ces changements motivés par l'aspect économique de la médecine. Nous allons devoir en prouver le bien-fondé. Des changements s'en viennent.

\section{Edward J. Harvey, MD}

Corédacteur en chef, fournal canadien de chirurgie.

Intérêts concurrents : E.J. Harvey est médecin chef chez Greybox Solutions; il est cofondateur et directeur de l'innovation médicale de NXTSens Inc, cofondateur et médecin chef de MY01 Inc. et cofondateur et directeur de Strathera Inc. Il bénéficie du soutien institutionnel de J \& J, DePuy Synthes, Stryker et Zimmer, et il fait partie du conseil d'administration de l'Orthopedic Trauma Association et de l'Association canadienne d'orthopédie.

DOI: $10.1503 /$ cjs.008419

\section{Références}

1. Flodgren G, Rachas A, Farmer AJ, et al. Interactive telemedicine: effects on professional practice and health care outcomes. Cochrane Database Syst Rev 2015;9:CD002098.

2. Vyas S, Murren-Boezem J, Solo-Josephson P. Analysis of a pediatric telemedicine program. Telemedicine and e-Health. Le 1er décembre 2018 [cyberpublication avant impression]. Accessible ici : https://doi.org/10.1089/tmj.2017.0281 (consulté le 6 mai 2019). 